\title{
Uso de ferramentas de avaliação para as doenças crônicas em pesquisas de enfermagem: revisão integrativa
}

\author{
Use of assessment tools for chronic disease in nursing research: integrative review
}

Uso de herramientas de evaluación para las enfermedades crónicas en investigación de enfermería: revisión integrativa

Wendy Julia Mariano Viante ${ }^{1}$, Maria de Fátima Mantovani ${ }^{2}$, Robson Giovani Paes ${ }^{2 \star}$, Marta Cossetin Costa $^{2}$, Thiago Nunes de Souza², Mahara Louíse Küchler².

\section{RESUMO}

Objetivo: Identificar as ferramentas de avaliação utilizadas em pesquisas de enfermagem nos adultos com doenças crônicas. Métodos: Revisão integrativa que seguiu seis etapas, com busca dos estudos primários na Biblioteca Virtual de Saúde e na National Library of Medicine, com os descritores "adulto", "doença crônica", "escalas", "instrumentos", "questionários", "cuidados de enfermagem", "adult", "chronic disease", "scale", "instrument", "questionnaire" e "nursing care". Os critérios de inclusão foram publicações de 2008 a 2018, em português, inglês e espanhol. Na busca inicial obtiveram-se 377 estudos, aplicou-se os critérios permanecendo 20, dos quais 15 compuseram a amostra final. Resultados: Sete estudos avaliaram as condições clínicas/emocionais, autoeficácia, sintomas e qualidade de vida; cinco mensuraram os benefícios das intervenções; e três construíram novas ferramentas. Dentre os instrumentos mais utilizados estavam o Medical Outcomes Study 36-item Short Form Health Survey, Hospital Anxiety and Depression Scale, Beck Depression Inventory e o Mini Exame do Estado Mental. Considerações finais: Considera-se que o emprego de ferramentas de avaliação deve ser estimulado desde a formação acadêmica, para a construção do raciocínio clínico, avaliação e planejamento do cuidado de enfermagem. As limitações referem-se aos critérios de inclusão e exclusão dos artigos, o recorte temporal e o número de base de dados pesquisada.

Palavras-chave: Doença crônica, Saúde do adulto, Cuidados de enfermagem, Pesos e medidas, Inquéritos e questionários.

\begin{abstract}
Objective: To identify the assessment tools used in nursing research in adults with chronic diseases. Methods: Integrative review that followed six stages, with a search for primary studies in the Virtual Health Library and in the National Library of Medicine, with the descriptors "adult", "chronic disease", "scale", "instrument", "questionnaire" and "nursing care". The inclusion criteria were from publications from 2008 to 2018, in Portuguese, English and Spanish. In the initial search, 377 studies were obtained, the criteria were applied, leaving 20, of which 15 made up the final sample. Results: Seven studies evaluated clinical-emotional conditions, self-efficacy, symptoms and quality of life; five measured the benefits of the interventions; and three built new tools. Among the most widely used instruments are the Medical Outcomes Study 36-item Short Form Health Survey, Hospital Anxiety and Depression Scale, Beck Depression Inventory and the Mini Mental State Exam. Final considerations: It is considered that the use of assessment tools should be encouraged from academic training, for the construction of clinical reasoning, evaluation and planning of nursing care. The limitations refer to the inclusion and exclusion criteria of the articles, the time frame and the number of databases consulted.
\end{abstract}

Keywords: Chronic disease, Adult health, Nursing care, Weights and measures, Surveys and questionnaires.

${ }^{1}$ Hospital de Clínicas do Paraná (HC-UFPR), Curitiba - PR.

²Universidade Federal do Paraná (UFPR), Curitiba - PR. *E-mail: robson.paes@ufpr.br 


\section{RESUMEN}

Objetivo: Identificar las herramientas de evaluación utilizadas en investigaciones de enfermería en los adultos con enfermedades crónicas. Métodos: Revisión integrativa que siguió seis etapas, con búsqueda de los estudios primarios en la Biblioteca Virtual de Salud e en la National Library of Medicine, con los descriptores "adult", "chronic disease", "scale", "instrument", "questionnaire" e "nursing care". Los criterios de inclusión fueron de publicaciones de 2008 a 2018, en portugués, inglés y español. En la búsqueda inicial se obtuvieron 377 estudios, se aplicaron los criterios, quedando 20, de los cuales 15 conformaron la muestra final. Resultados: Siete estudios evaluaron condiciones clínico-emocionales, autoeficacia, síntomas y calidad de vida; cinco midieron los beneficios de las intervenciones; y tres construyeron nuevas herramientas. Entre los instrumentos más utilizados se encuentran el Medical Outcomes Study 36-item Short Form Health Survey, Hospital Anxiety and Depression Scale, Beck Depression Inventory y el Mini Examen del Estado Mental. Consideraciones finales: Se considera que se debe fomentar el uso de herramientas de evaluación desde la formación académica, para la construcción del razonamiento clínico, evaluación y planificación de los cuidados de enfermería. Las limitaciones se refieren a los criterios de inclusión y exclusión de los artículos, el marco temporal y el número de bases de datos consultadas.

Palabras clave: Enfermedad crónica, Salud del adulto, Atención de enfermería, Pesos y medidas, Encuestas y cuestionarios.

\section{INTRODUÇÃO}

As doenças crônicas não transmissíveis (DCNTs) têm alta prevalência na população mundial, possuem características multifatoriais, determinantes biológicos e socioculturais. Em geral apresentam início gradual, cursos clínicos longos e incertos, com períodos, de agudização, logo podem causar complicações e incapacidades (BRASIL; MS, 2013a; BRASIL; MS, 2014). Uma vez que apresentam altas taxas de morbimortalidade.

Para reduzir os agravos das DCNTs, estratégias são recomendadas para auxiliar na identificação de risco, prevenção de complicações, diminuição das taxas de reinternação e dos custos do tratamento. $O$ uso de tecnologias como as ferramentas de avaliação, facilitam o cuidado e as ações de educação em saúde, por meio da mensuração de adesão ao tratamento, do suporte para o gerenciamento dos cuidados e da qualidade de vida (MANTOVANI MF, et al., 2015).

As ferramentas de avaliação ou instrumentos, escalas e questionários, são indicadores de medidas de diversas naturezas como as prognósticas, funcionais, técnicas, preditivas entre outras. Os instrumentos podem ser específicos ou genéricos e tendem a comparar condições clínicas, epidemiológicas, terapêuticas, registrando a presença de gravidades, sintomas ou deficiências (MCDOWELL I, 2006).

As escalas são compostas por itens de avaliação definidos, cujas medidas podem variar de acordo com a aplicação e o julgamento do profissional avaliador. Os questionários contêm elementos pré-definidos pelo autor, com argumentos de abordagem subjetiva e com diferentes maneiras de aplicação; sua mensuração coletiva se dá por meio da consistência das respostas dos participantes (MCDOWELL I, 2006).

No desempenho das ações de enfermagem, essas ferramentas são consideradas tecnologias leves e duras, pois envolvem desde a relação entre o profissional e a pessoa, até a utilização de equipamentos, métodos e técnicas, os quais são aliados aos saberes estruturados que alicerçam as profissões (ALMEIDA Q; FÓFANO GA, 2016), além disso, o uso dessas tecnologias nas consultas de enfermagem ajudam na identificação dos problemas e podem aumentar a resolutividade quando as metas são pactuadas em conjunto com a pessoa.

Uma estratégia importante para o manejo adequado das DCNTs é a estratificação de risco, que leva em consideração a severidade da condição crônica estabelecida e a capacidade de autocuidado para ajustar o plano de ações para controle da doença e redução de complicações (BRASIL MS, 2014).

O Escore de Framingham é uma das ferramentas mais conhecidos para estratificação das DCNTs, pois pode predizer o risco de eventos cardiovasculares nos próximos 10 anos, baseando-se em níveis pressóricos, 
exames laboratoriais, hábitos de vida e características individuais (BRASIL MS, 2013). Dessa forma, pode-se inferir que a estratificação de risco e, por consequência, as orientações, as prescrições de cuidados de enfermagem e o gerenciamento de caso são peças-chave para a melhora da condição crônica (HONORATO DZS, et al., 2015).

Seguindo as recomendações do Ministério da Saúde para a prevenção das complicações das DCNTs, o uso de ferramentas de avaliação, têm auxiliado a enfermagem na condução dos cuidados, acompanhamento, gerenciamento e tomada de decisão (SILVA ATM, et al., 2017).

Frente a isso, torna-se fundamental a identificação das ferramentas utilizadas nas DCNTs, para compreender como os resultados destas, têm sido usados na prática de enfermagem. Diante do exposto, e, sobretudo pelo potencial que as tecnologias leve-duras possuem em aprimorar os cuidados em saúde, o objetivo deste trabalho foi identificar as ferramentas de avaliação utilizadas em pesquisas de enfermagem nos adultos com doenças crônicas não transmissíveis.

\section{MÉTODOS}

Trata-se de uma revisão integrativa da literatura, fundamentada em seis etapas: 1) elaboração da questão da pesquisa; 2) estabelecimento dos critérios de inclusão/exclusão de estudos; 3) categorização dos estudos; 4) avaliação dos estudos incluídos; 5) interpretação dos resultados; 6) apresentação dos resultados e síntese do conhecimento (MENDES KDS, et al., 2008).

A pergunta norteadora da pesquisa foi elaborada com o auxílio de uma adaptação da estratégia PICO, substituída por PCC que representa um acrônimo das palavras Paciente, Conceito e Contexto, a saber P: adultos com doença crônica; C: escalas, instrumentos ou questionários; e C: o uso de escalas, instrumentos ou questionários em pesquisas de enfermagem nos adultos com DCNTs. Sendo assim, a pergunta de pesquisa foi: quais são as ferramentas de avaliação utilizadas em pesquisas de enfermagem nos adultos com doenças crônicas não transmissíveis?

Os critérios de inclusão foram: estudos que abordassem a utilização de escalas, questionários ou instrumentos em pacientes adultos a partir de 18 anos com DCNTs, associados aos cuidados de enfermagem; publicados entre 2008 e 2018, com texto completo disponível nos idiomas inglês, ou português, ou espanhol. Os critérios de exclusão foram: estudos com crianças, cuidadores de pessoas com ou sem DCNTs, revisão integrativas, escopo, mini ou sistemáticas, carta ao editor e consenso de especialistas.

A busca dos artigos foi realizada no Portal Regional da Biblioteca Virtual em Saúde (BVS) e na National Library of Medicine (PubMed), as quais reúnem diversas fontes de informação em saúde, como as bases de dados da Literatura Latino-americana e do Caribe em Ciências da Saúde (LILACS) e Medical Literature Analysis and Retrieval System Online (Medline), além de publicações do Ministério de Saúde do Brasil. A estratégia de busca consistiu em três etapas para cada base de dados (Quadro 1).

Quadro 1 - Estratégia de busca utilizada no portal da BVS, Curitiba, PR, Brasil, 2018.

\begin{tabular}{|c|c|c|c|}
\hline \multirow{2}{*}{$\begin{array}{c}\text { Etapa } \\
1\end{array}$} & BVS & $\begin{array}{c}\text { (tw:(adulto)) AND (tw:(“doença crônica”)) AND (tw:(escala)) } \\
\text { AND (tw:(“cuidados de enfermagem”)) }\end{array}$ & \multirow{6}{*}{$\begin{array}{l}\text { Filtros: publicados } \\
\text { entre } 2008 \text { a } \\
\text { 2018; idiomas } \\
\text { inglês, português } \\
\text { e espanhol; texto } \\
\text { completo }\end{array}$} \\
\hline & PubMed & $\begin{array}{c}\text { Adult[Mesh] AND "chronic Disease"[Mesh] AND Scale[Mesh] } \\
\text { AND "nursing care"[Mesh] }\end{array}$ & \\
\hline \multirow{2}{*}{$\begin{array}{c}\text { Etapa } \\
2\end{array}$} & BVS & $\begin{array}{c}\text { (tw:(adulto)) AND (tw:(“doença crônica”)) AND } \\
\text { (tw:(instrumentos)) AND (tw:(“cuidados de enfermagem”)) }\end{array}$ & \\
\hline & PubMed & $\begin{array}{c}\text { Adult [Mesh] AND "chronic disease" [Mesh] AND instrument } \\
\text { [Mesh] AND "nursing care" [Mesh] }\end{array}$ & \\
\hline \multirow{2}{*}{$\begin{array}{c}\text { Etapa } \\
3\end{array}$} & BVS & $\begin{array}{c}\text { (tw:(adulto)) AND (tw:(“doença crônica”)) AND } \\
\text { (tw:(questionários)) AND (tw:(“cuidados de enfermagem”)) }\end{array}$ & \\
\hline & PubMed & $\begin{array}{c}\text { Adult [Mesh]AND "chronic disease" [Mesh] AND questionnaire } \\
\text { [Mesh] AND"nursing care" [Mesh] }\end{array}$ & \\
\hline
\end{tabular}

Fonte: Viante WJM, et al., 2020. 
Após a aplicação dos filtros foi realizada a leitura dos títulos e resumos, aplicando-se os critérios de inclusão e exclusão; elegidos 20 estudos para leitura na íntegra. Cinco artigos não contemplavam a proposta do assunto, sendo 15 selecionados para a sumarização da revisão. Utilizado o fluxograma do The Preferred Reporting Systematic Reviews and Meta-Analyses of Studies (PRISMA) (GALVÃO TF, et al., 2015) para apresentação da busca (Figura 2).

Figura 2 - Fluxograma PRISMA para captação, seleção e inclusão de estudos para a revisão integrativa, Curitiba, Paraná, Brasil, 2018.
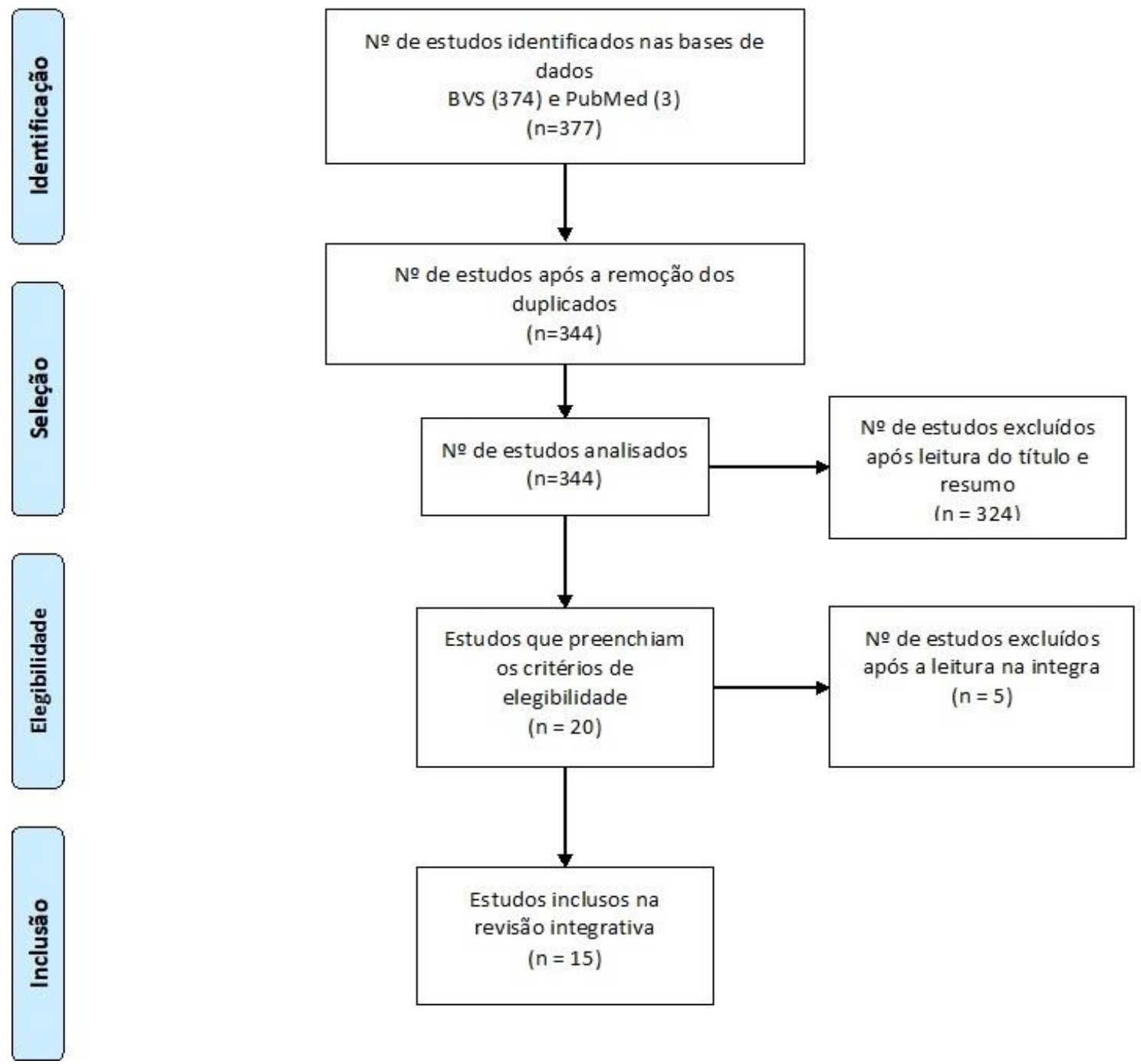

Fonte: Viante WJM, et al., 2020. Adaptado de Galvão TF, et al., 2015.

Os dados referentes aos resultados dos estudos foram apresentados em um quadro contendo: autores, ano, local, objetivos e a utilização de ferramentas de avaliação. Os artigos foram identificados pelo prefixo A1 a A15, e procedeu-se a análise descritiva dos dados.

\section{RESULTADOS}

Foram encontrados 377 estudos na busca inicial, após removido os duplicados e realizado a leitura dos títulos e resumos conforme os critérios estabelecidos. Os 20 artigos elegidos foram lidos na integra, sendo selecionados 15 para a revisão integrativa da literatura. Dentre as escalas, instrumentos ou questionários mais utilizados, estão o Medical Outcomes Study 36-item Short Form Health Survey (SF-36), utilizado em cinco artigos; Hospital Anxiety and Depression Scale (HADS), utilizada em três artigos; Beck Depression Inventory (BDI), utilizado em dois artigos; e Mini Exame do Estado Mental (MEEM), utilizada em dois artigos. Os demais instrumentos não foram utilizados por mais de um autor encontrado nessa revisão. A síntese dos resultados é apresentada no Quadro 2. 


\section{Revista Eletrônica Acervo Saúde / Electronic Journal Collection Health | ISSN 2178-2091}

Quadro 2 - Apresentação dos estudos incluídos na revisão integrativa, Curitiba, Paraná, Brasil, 2018.

\begin{tabular}{|c|c|c|}
\hline Prefixo, autores, ano e local & Objetivos & Ferramentas \\
\hline $\begin{array}{l}\text { A1. SOARES DA, et al., } 2008 . \\
\text { Brasil. }\end{array}$ & $\begin{array}{c}\text { Identificar o nível de qualidade de vida das pessoas com } \\
\text { insuficiência cardíaca; o grau da insuficiência; a opinião } \\
\text { sobre o seu estado de saúde atual quando comparada ao } \\
\text { ano anterior. }\end{array}$ & SF-36 \\
\hline $\begin{array}{l}\text { A2. SUN V, et al., } 2008 . \\
\text { Estados Unidos. }\end{array}$ & $\begin{array}{c}\text { Descrever as preocupações com sintomas de pessoas com } \\
\text { carcinoma hepatocelular e câncer de pâncreas; explorar o } \\
\text { efeito dos sintomas na qualidade de vida. }\end{array}$ & $\begin{array}{c}\text { Functional Assessment of Cancer Therapy- Hepatobiliary (FACT- } \\
\text { Hep); FACIT-Spirituality Subscale (FACIT-Sp-12). }\end{array}$ \\
\hline $\begin{array}{l}\text { A3. MADAR H e BAL-TAL Y, } \\
\text { 2009. Israel. }\end{array}$ & $\begin{array}{c}\text { Examinar fatores (gravidade, duração da doença, } \\
\text { autoridade confiável, apoio social e educação) que podem } \\
\text { influenciar o nível de incerteza e estresse de pessoas em } \\
\text { diálise peritoneal. }\end{array}$ & $\begin{array}{l}\text { Mishel's Uncertainty in Illness Scale (MUIS); Tel Aviv Social } \\
\text { Support Instrument; Versão adaptada da Epistemic Authority Scale } \\
\text { (EAS); Patient level of stress score (de MADAR HE BAL-TAL Y). }\end{array}$ \\
\hline $\begin{array}{l}\text { A4. LINDBERG M, et al., } \\
\text { 2010. Suécia. }\end{array}$ & $\begin{array}{l}\text { Determinar subgrupos definíveis em uma amostra de } \\
\text { pessoas em hemodiálise, quanto a autoeficácia, estilo de } \\
\text { atenção e sintomatologia depressiva; comparar se o ganho } \\
\text { de peso interdialítico varia entre grupos com diferentes } \\
\text { perfis cognitivos. }\end{array}$ & $\begin{array}{c}\text { Fluid Intake Appraisal Inventory (FIAI); Threatening Medical } \\
\text { Situation Inventory (TMSI); Center for Epidemiologic Studies } \\
\text { Depression scale (CES-D) short version. }\end{array}$ \\
\hline $\begin{array}{l}\text { A5. LIAO MN, et al., } 2012 . \\
\text { Taiwan. }\end{array}$ & $\begin{array}{l}\text { Explorar mudanças longitudinais na angústia dos sintomas } \\
\text { em pessoas recém-diagnosticados com câncer de mama e } \\
\text { fatores que preveem alterações desde o momento do } \\
\text { diagnóstico até } 4 \text { meses de tratamento. }\end{array}$ & $\begin{array}{c}\text { State-Trait Anxiety Inventory (STAI), Chinese version; Social } \\
\text { Support Scale-modified (SSS-m), Chinese version; Symptom } \\
\text { Distress Scale-Modified for Breast Cancer (SDS-mbc), Chinese } \\
\text { version; Supportive Care Needs Survey-Short Form (SCNS-SF34), } \\
\text { Chinese version. }\end{array}$ \\
\hline $\begin{array}{l}\text { A6. WOOD-BAKER R, et al., } \\
\text { 2012. Tasmânia. }\end{array}$ & $\begin{array}{l}\text { Investigar o efeito de um programa de aumento dos } \\
\text { comportamentos de autogestão prestados por enfermeiros } \\
\text { comunitários de saúde, em comparação com os cuidados } \\
\text { habituais, sobre a qualidade de vida relacionada à saúde e } \\
\text { a utilização da atenção à saúde em pessoas com doença } \\
\text { pulmonar obstrutiva crônica após internação. }\end{array}$ & $\begin{array}{c}\text { HADS; Stanford self-efficacy questionnaire; SF-36; Geriatric Index } \\
\text { of Comorbidity; MRC dyspnea score. }\end{array}$ \\
\hline $\begin{array}{l}\text { A7. MULDER M, et al., } 2013 . \\
\text { Holanda. }\end{array}$ & $\begin{array}{c}\text { Desenvolver e validar um instrumento de triagem para os } \\
\text { enfermeiros avaliarem o medo do cateter em pessoas em } \\
\text { diálise. }\end{array}$ & $\begin{array}{c}\text { HADS; Dialysis Fear of Injection Questionnaire (D-FIQ) versão } \\
\text { adaptada do Diabetes Fear of Injection and Self-Testing } \\
\text { Questionnaire (D-FISQ). }\end{array}$ \\
\hline
\end{tabular}




\section{Revista Eletrônica Acervo Saúde / Electronic Journal Collection Health | ISSN 2178-2091}

\begin{tabular}{|c|c|c|}
\hline Prefixo, autores, ano e local & Objetivos & Ferramentas \\
\hline $\begin{array}{l}\text { A8. VOORN VMA, et al., } 2013 . \\
\text { Holanda. }\end{array}$ & $\begin{array}{l}\text { Investigar a dor, função física, qualidade de vida } \\
\text { relacionada à saúde em pessoas com osteoartrite do } \\
\text { quadril ou joelho após uma intervenção multidisciplinar. }\end{array}$ & $\begin{array}{l}\text { SF-36; Euro Quality of Life-5 (EQ-5D); Intermittent and constant } \\
\text { osteoarthritis pain questionnaire (ICOAP); Hip disability and } \\
\text { osteoarthritis outcome score (HOOS); Knee injury and osteoarthritis } \\
\text { outcomes score (KOOS); Patient satisfaction questionnaire. }\end{array}$ \\
\hline $\begin{array}{l}\text { A9. AL-GAMAL E, } 2014 . \\
\text { Jordânia. }\end{array}$ & $\begin{array}{l}\text { Examinar as relações entre ansiedade, depressão e } \\
\text { qualidade de vida de jordanianos com doença pulmonar } \\
\text { obstrutiva crônica e seus cônjuges. }\end{array}$ & $\begin{array}{l}\text { HADS; Quality of Life Inventory - Pulmonary version (QLI-P); } \\
\text { Quality of Life Inventory - Generic version (QLI-G). }\end{array}$ \\
\hline $\begin{array}{l}\text { A10. DETROYER E, et al., } \\
\text { 2014. Bélgica. }\end{array}$ & $\begin{array}{c}\text { Determinar a validade diagnóstica e simultânea, a } \\
\text { consistência interna e a simpatia do usuário com a Delirium } \\
\text { Observation Screening Scale administrada por enfermeiros } \\
\text { de cabeceira na unidade de cuidados paliativos. }\end{array}$ & $\begin{array}{c}\text { Delirium Observation Screening Scale; Confusion Assessment } \\
\text { Method (CAM); Delirium Index (DI). }\end{array}$ \\
\hline $\begin{array}{l}\text { A11. LUCAS R, et al., } 2014 . \\
\text { Reino Unido. }\end{array}$ & $\begin{array}{c}\text { Explorar o efeito do contato de enfermeiras especialistas } \\
\text { em insuficieincia cardíaca nas crenças, humor e qualidade } \\
\text { de vida. }\end{array}$ & $\begin{array}{c}\text { Minnesota Living with Heart Failure Questionnaire (MLwHF);HADS; } \\
\text { IIIness Perception Questionnaire (IPQ-R); Treatment } \\
\text { Representations Inventory (TRI). }\end{array}$ \\
\hline $\begin{array}{l}\text { A12. PAUDEL K, et al., } 2015 . \\
\text { Reino Unido. }\end{array}$ & $\begin{array}{c}\text { Determinar se o padrão de moradia, a técnica de troca de } \\
\text { Diálise Peritoneal ou a motivação podem ser riscos } \\
\text { modificáveis para peritonite; explorar a relação entre falta } \\
\text { de motivação e depressão. }\end{array}$ & $\begin{array}{c}\text { Questionário adaptado do Apathy Evaluation Score (AES); Patient } \\
\text { Health Questionnaire (PHQ-9) for Depression score. }\end{array}$ \\
\hline $\begin{array}{l}\text { A13. TSAI SH, et al., } 2015 . \\
\text { Taiwan. }\end{array}$ & $\begin{array}{l}\text { Examinar a eficácia de um programa de treinamento } \\
\text { respiratório orientado por enfermeiras na redução de } \\
\text { sintomas depressivos e na melhoria da qualidade de vida, } \\
\text { do sono e da saúde em pessoas em hemodiálise. }\end{array}$ & $\begin{array}{c}\text { Beck Depression Inventory-II Chinese version; Pittsburgh Sleep } \\
\text { Quality Index (PSQI);Medical Outcomes Study 36-item Short Form } \\
\text { Health Survey. }\end{array}$ \\
\hline $\begin{array}{l}\text { A14. ULBRICH EM, } 2015 . \\
\text { Brasil. }\end{array}$ & $\begin{array}{c}\text { Elaborar escala preditiva de determinantes para } \\
\text { complicações em pessoas com hipertensão arterial } \\
\text { sistêmica e ações para o gerenciamento de cuidado em } \\
\text { enfermagem. }\end{array}$ & $\begin{array}{l}\text { MEEM; Escala de apoio social adaptada transculturalmente da } \\
\text { Medical Outcomes Study; BDI; Inventário de ansiedade de Beck; } \\
\text { SF-36; Brief Medication Questionnaire. }\end{array}$ \\
\hline $\begin{array}{l}\text { A15. ZAKRISSON AB, et } \\
\text { al.,2016. Suécia. }\end{array}$ & $\begin{array}{l}\text { Investigar os efeitos de um programa de reabilitação } \\
\text { pulmonar multidisciplinar sobre a capacidade funcional, } \\
\text { qualidade de vida e frequência de exacerbação de } \\
\text { sintomas ao longo de três anos entre as pessoas com } \\
\text { doença pulmonar obstrutiva crônica. }\end{array}$ & $\begin{array}{l}\text { Six-minute walking-test (6MWT) (capacidade functional); Clinical } \\
\text { COPD Questionnaire (CCQ). }\end{array}$ \\
\hline
\end{tabular}

Fonte: Viante WJM, et al., 2020. 
Dentre os estudos analisados, percebeu-se que sete estudos utilizaram escalas ou instrumentos para avaliar aspectos clínicos ou emocionais, autoeficácia, sintomas ou qualidade de vida de pessoas com DCNTs (SOARES DA, et al., 2008; SUN V, et al., 2008; MADAR H e BAR-TAL Y, 2009; LINDBERG M, et al., 2010; LIAO MN, et al., 2015; AL-GAMAL E, 2014; PAUDEL K, et al., 2015).

Cinco estudos aplicaram os instrumentos ou escalas com o objetivo de subsidiar ou avaliar a eficácia de uma intervenção baseada em cuidados de enfermagem direcionada as pessoas com DCNTs (WOOD-BAKER R, et al., 2012; VOORN VMA, et al., 2013; LUCAS R, et al., 2014; TSAI SH, et al., 2015; ZAKRISSON AB, et al., 2016).

Três estudos aplicaram instrumentos durante a coleta de dados para a construção de outro instrumento relacionado à assistência em saúde (MULDER M, et al., 2013; DETROYER E, et al., 2014; ULBRICH EM, 2015). A autora do A14, desenvolveu uma escala para predição de complicações da hipertensão arterial sistêmica. Os instrumentos utilizados na coleta de dados foram aplicados para selecionar as variáveis que causavam impacto no desenvolvimento de complicações da doença (ZAKRISSON AB, et al., 2016).

No A7 os autores desenvolveram e validaram um instrumento de triagem utilizado para avaliar o medo de injeção em pessoas que realizam diálise, o Dialysis Fear of Injection Questionnaire, desenvolvido com base em outro instrumento, o da Diabetes Fear of Injection and Self-Testing Questionnaire (MULDER M, et al., 2013).

Já no A10 os autores pretendiam confirmar a validade diagnóstica e concorrente, além da consistência interna e a facilidade de uso do Delirium Observation Screening Scale, que pode ser utilizada por enfermeiros em unidades de cuidados paliativos (DETROYER E, et al., 2014).

\section{DISCUSSÃO}

Observou-se que nove estudos realizaram a mensuração da qualidade de vida dos participantes com DCNTs. A utilização de instrumentos para mensurar a qualidade de vida possibilita compreender e avaliar as condições econômicas, sociais e de saúde da pessoa e da coletividade, fornecendo informações relevantes para o planejamento das intervenções. Ainda contribui para a humanização e a efetividade dos serviços de saúde (RÔLA CVS, et al., 2018).

Dentre as variáveis mais utilizadas nos instrumentos de qualidade de vida estão as de domínio físico, como dor, fadiga e limitações; domínio psicológico, envolvendo a percepção sobre a saúde, ansiedade, autoestima, depressão e imagem corporal; relações sociais, como o apoio familiar e relações interpessoais; independência, abordando questões como as atividades do cotidiano, a mobilidade e a capacidade para o trabalho; e por fim, noções referentes ao bem estar e saúde mental, aspectos que poderiam ser utilizados para o cuidado de enfermagem (CARNEIRO ACC, et al., 2018; SOUZA AR, et al., 2018).

Foram encontrados, nove instrumentos de avaliação de qualidade de vida, sendo três de uso genérico e os demais específicos para doença pulmonar obstrutiva crônica, insuficiência cardíaca, osteoartrite, câncer hepatobiliar e espiritualidade. O instrumento genérico mais citado foi o Medical Outcomes Study 36-item Short Form Health Survey, resultado que corroborou com uma revisão sistemática realizada no Brasil em 2018, a qual teve como objetivo mostrar os principais instrumentos validados de qualidade de vida para jovens e idosos (RÔLA CVS, et al., 2018).

O Medical Outcomes Study 36-item Short Form Health Survey é amplamente utilizado devido a sua brevidade e abrangência (WARE JE e SHERBOURNE CD, 1992; RAJATI F, et al., 2016). Nesta revisão podese observar a utilização do Medical Outcomes Study 36-item Short Form Health Survey nas diferentes doenças crônicas como na insuficiência cardíaca (SOARES DA, et al., 2008), doença pulmonar obstrutiva crônica (WOOD-BAKER R, et al., 2012), doenças reumáticas (VOORN VMA, et al., 2013), doença renal crônica em terapia substitutiva (TSAI SH, et al., 2015) e ainda como subsídio para construção de uma nova escala (ULBRICH EM, 2015).

O Medical Outcomes Study 36-item Short Form Health Survey é um questionário composto por 36 itens que avaliam oito domínios em saúde, sendo eles: a percepção em saúde (1); limitações em atividades diárias 
por problemas de saúde (2); limitações em atividades regulares por problemas de saúde física (3) ou emocional (4); dor corporal (5); saúde mental (6); energia e emoções (7); e limitações nas atividades sociais devido a problemas físicos ou emocionais (8) (WARE JE e SHERBOURNE CD, 1992).

Por tratar-se de um instrumento versátil, o Medical Outcomes Study 36-item Short Form Health Survey pode ser aplicado por telefonema, autoadministração ou pessoalmente em entrevista. Nas pessoas com doenças crônicas em internamento hospitalar por longo período de tempo, este questionário pode ser complementado com outros itens voltados para a doença (WARE JE e SHERBOURNE CD, 1992). Permitindo reconhecer as alterações nas diversas dimensões da pessoa com doença crônica.

Uma pesquisa observacional prospectiva realizada na Espanha, teve como objetivo determinar os níveis de qualidade de vida e de ansiedade nas mulheres antes e após o tratamento de câncer de mama. Participaram do estudo 339 mulheres que durante o diagnóstico obtiveram maiores pontuações nos domínios de função física, função funcional e imagem corporal, e baixa pontuação nas perspectivas futuras e prazer sexual. Após o tratamento apresentaram menor pontuação da qualidade de vida nas funções física, funcional, imagem corporal, dificuldades financeiras, sintomas de fadiga, dor e dispneia. Mulheres com níveis de escolaridade mais alto obtiveram melhores pontuações da qualidade de vida antes e após o tratamento (VILLAR RR, et al., 2017).

Com relação aos níveis de ansiedade, $45 \%$ das participantes tinham níveis graves de ansiedade no momento do diagnóstico. Por meio da análise de regressão logística multivariável e independente, observouse que aquelas com união consensual, sem emprego formal, que apresentavam edema de mama no momento do diagnóstico e que utilizavam medicamentos ansiolíticos, foram as que mais apresentaram níveis graves de ansiedade comparadas as mulheres solteiras, com emprego formal e sem alterações mamárias (VILLAR $\mathrm{RR}$, et al., 2017). A utilização desses instrumentos permitiu avaliar as questões relativas aos impactos causados pela doença crônica na qualidade de vida pela ansiedade.

Nesta revisão, o instrumento mais citado para mensurar a ansiedade foi o Hospital Anxiety and Depression Scale, aplicado para pessoas com doenças renais crônicas e doença pulmonar obstrutiva crônica (WOODBAKER R, et al. 2012; MULDERM, et al., 2013; AL-GAMAL E, 2014). Este instrumento é auto-aplicável, e pode ser utilizado em ambientes de atendimento ambulatorial e hospitalar, em contextos não psiquiátricos, para detectar estados de ansiedade e depressão (AYIS AS, et al., 2018).

O Hospital Anxiety and Depression Scale contém duas sub-escalas, uma para ansiedade e outra para depressão, e cada sub-escala é compostas de sete itens. Cada item contém quatro opções de respostas, as quais possuem uma pontuação que varia de 0 a 3 , de modo que cada escala pode ter um escore final variando de 0 a 21 pontos. Valores de pontuação entre 0 e 7 são considerados como um intervalo normal do estado emocional; valores entre 8 e 10 sugerem a presença de um transtorno de humor; e valores acima de 11 indicam possíveis casos de ansiedade ou depressão (SNAITH RP, 2003).

Fato observado em um estudo realizado com pessoas com doença renal crônica, que avaliou a presença de ansiedade e depressão utilizando a Hospital Anxiety and Depression Scale e relacionou estes fatores com a qualidade de vida. Os resultados demonstraram que escores mais altos nas sub-escalas da Hospital Anxiety and Depression Scale estão correlacionados com menor índice de qualidade de vida, o que evidencia 0 impacto da doença crônica e do curso do tratamento na vida das pessoas (OTTAVIANI AC, et al., 2016).

Outros instrumentos que avaliam depressão e ansiedade, os quais também foram utilizados em estudos encontrados nesta revisão integrativa são o State-Trait Anxiety Inventory, Inventário de ansiedade de Beck e Beck Depression Inventory. O Beck Depression Inventory é um questionário auto-aplicável de ampla utilização em estudos, bem como na prática clínica. Possui 21 itens, cada um contendo quatro afirmações com valores entre 0 e 3 , abordando sentimentos como tristeza, pessimismo, crises de choro, culpa, distúrbios do sono e do apetite, irritabilidade, retração social, entre outros (BECK AT, et al., 1961).

A pontuação final difere dependendo da amostra em que o questionário foi aplicado. Em amostras com transtorno afetivo, recomenda-se considerar os seguintes pontos de corte: menor que $10=$ sem depressão ou depressão mínima; de 10 a 18 = depressão leve a moderada; de 19 a 29 = depressão, de moderada a 
grave; de 30 a 63 = depressão grave (BECK AT, et al., 1987). Para amostras sem diagnóstico clínico anterior, recomenda-se considerar escores acima de 15 para detectar disforia e considerar escores acima de 20 como depressão, preferencialmente com diagnóstico clínico concomitante (KENDALL PC, et al., 1987).

Uma pesquisa transversal realizada no estado do Paraná teve como objetivo investigar a relação de depressão e qualidade de vida em 387 adultos com hipertensão arterial, utilizando o Medical Outcomes Study 36-item Short Form Health Survey e o Beck Depression Inventory. A depressão diagnosticada por médico autorrelatada estava presente em 168 participantes, os quais também obtinham médias baixas de qualidade de vida nos domínios de dor, vitalidade, estado geral de saúde e aspecto. Quando comparados os participantes com e sem depressão, os autores identificaram que as médias dos domínios foram significativamente menores para os que possuíam depressão, com destaque para os aspectos emocionais, a saúde mental e vitalidade (MANTOVANI MF, et al., 2017).

A avaliação da ansiedade e depressão em pessoas com DCNTs, é relevante para o curso do tratamento, pois altos níveis de ansiedade e depresssão causam impacto significativo na qualidade de vida. Além disso, quando a pessoa possui baixo conhecimento sobre a doença, expectativas, tratamento e complicações, a ansiedade pode ser elevada, devido ao enfrentamento do desconhecido. Da mesma forma, em situações de agravamento ou complicação das DCNTs, podem ocorrer sintomas depressivos e redução da adesão ao tratamento, fator que eleva a morbimortalidade (VILLAR RR, et al, 2017; SOUZA FTZ, OLIVEIRA JHA, 2017; MANTOVANI MF, et al., 2017). A utilização destes instrumentos na prática profissional, podem auxiliar na detecção dos transtornos emocionais entre outros (SNAITH RP, 2003).

O Mini Exame do Estado Mental foi utilizado em dois estudos, sendo esse um teste para identificar alterações cognitivas. É composto por duas etapas: a primeira é estruturada em perguntas que necessitam apenas de respostas verbais, abrangendo domínios como orientação, memória e atenção, e a pontuação máxima nesta etapa é 21. Na segunda etapa, são necessárias habilidades de leitura e escrita, e os domínios avaliados são a capacidade de nomear objetos, seguir comandos verbais e escritos, escrever uma frase espontaneamente, e copiar a figura de um polígono complexo, totalizando nove pontos (FOLSTEIN MF, et al., 1975).

Ao todo, há 11 questões, a pontuação máxima no Mini Exame do Estado Mental é 30, e o teste não precisa ser cronometrado. Devido a sua fácil aplicação em um curto espaço de tempo (5 a 10 minutos), o Mini Exame do Estado Mental é amplamente utilizado em estudos, principalmente quando envolve participantes idosos (FOLSTEIN MF, et al., 1975). No Mini Exame do Estado Mental os resultados apresentam diferenças decorrentes do nível de escolaridade dos participantes, sendo o corte de 13 pontos para pessoas sem escolaridade, 18 para baixa/média e 26 para alta escolaridade (ULBRICH EM, 2015).

Observa-se que as ferramentas encontradas nos estudos foram utilizadas para avaliar as pessoas com doenças crônicas, subsidiar intervenções e para contribuir na produção de uma nova ferramenta. As limitações desse estudo, referem-se aos critérios de inclusão e exclusão dos artigos, a limitação temporal e o número de base de dados pesquisada.

\section{CONSIDERAÇÕES FINAIS}

O objetivo de nosso estudo foi alcançado, pois evidenciamos o uso de escalas, instrumentos e questionários em pesquisas de enfermagem na investigação da qualidade de vida, ansiedade, depressão, autoeficácia, aspectos clínicos e psicológicos das pessoas com DCNTs. A maioria dessas ferramentas dão pistas para subsidiar os cuidados de enfermagem e/ou avaliar o efeito dos cuidados por meio de diagnóstico situacional. Dessa forma, acredita-se que o incentivo a utilização de escalas, instrumentos e questionários nas pesquisas de enfermagem motivem acadêmicos e enfermeiros para incorporação dessas ferramentas no planejamento da assistência, na construção do conhecimento e do raciocínio clínico associado às tecnologias de cuidado.

\section{AGRADECIMENTOS E FINANCIAMENTO}

O presente estudo foi realizado com o apoio da Coordenação de Aperfeiçoamento de Pessoal de Nível Superior - Brasil (CAPES) - código de financiamento 001, pelo Conselho Nacional de Desenvolvimento Científico e Tecnológico (CNPq) e pelo Tesouro Nacional (TN). 


\section{REFERÊNCIAS}

1. AL-GAMAL E. Quality of life, anxiety and depression among patients with chronic obstructive pulmonary disease and their spouses. Issues in Mental Health Nursing, 2014; 35(10): 761-767.

2. ALMEIDA Q, FÓFANO GA. Tecnologias leves aplicadas aos cuidados de enfermagem na unidade de terapia intensiva: uma revisão de literatura. HU Revista, 2016; 42(3): 191-196.

3. AYIS SA, et al. Evaluation of the hospital anxiety and depression scale (HADS) in screening stroke patients for symptoms: item response theory (IRT) analysis. Journal of Affective Disorders, 2018; 228: 33-40.

4. BECK AT, et al. An inventory for measuring depression. Archives of General Psychiatry, 1961; 4(6): 561-571.

5. BECK AT, et al. Psychometric properties of the Beck Depression Inventory: Twenty-five years of evaluation. Clinical Psychology Review, 1987; 8(1): 77-100.

6. BRASIL. Ministério da Saúde (MS). Diretrizes para o cuidado das pessoas com doenças crônicas nas redes de atenção à saúde e nas linhas de cuidado prioritárias. Brasília: MS; 2013a.

7. BRASIL. Ministério da Saúde (MS). Estratégias para o cuidado da pessoa com doença crônica: hipertensão arterial sistêmica. Brasília: MS; 2013b

8. BRASIL. Ministério da Saúde (MS). Estratégias para o cuidado da pessoa com doença crônica. Brasília: MS; 2014.

9. CARNEIRO ACC, et al. Questionários de qualidade de vida relacionada à saúde na doença pulmonar obstrutiva crônica (DPOC): revisão de literatura. Revista Internacional de Educação e Saúde, 2018; 2(1): 93-103.

10. DETROYER E. et al. Detection of delirium in palliative care unit patients: a prospective descriptive study of the Delirium Observation Screening Scale administered by bedside nurses. Palliative Medicine, 2014; 28(1): 79-86.

11. FOLSTEIN MF, et al. "mini-mental state": a practical method for grading the cognitive state of patients for the children. Journal of Psychiatric Research, 1975; 12(3): 189-198.

12. GALVÃO TF, et al. Principais itens para relatar revisões sistemáticas e meta-análises: a recomendação PRISMA. Epidemiologia e Serviços de Saúde, 2015; 24: 335-342.

13. HONORATO DZS, et al. O uso de tecnologias em saúde na consulta: uma análise reflexiva. Revista Interdisciplinar, 2015; 8(1): 234-239.

14. KENDALL PC, et al. Issues and recommendations regarding use of the Beck Depression Inventory. Cognitive Therapy and Research, 1987; 11(3): 289-299.

15. LIAO MN, et al. Change and predictors of symptom distress in breast cancer patients following the first 4 months after diagnosis. Journal of the Formosan Medical Association, 2015; 114(3): 246-253.

16. LINDBERG M, et al. Subgroups of haemodialysis patients in relation to fluid intake restrictions: a cluster analytical approach. Journal of Clinical Nursing, 2010; 19(21-22): 2997-3005.

17. LUCAS R, et al. The effect of heart failure nurse consultations on heart failure patients' illness beliefs, mood and quality of life over a six-month period. Journal of Clinical Nursing, 2014; 24(1-2): 256-265.

18. MADAR H, BAR-TAL Y. The experience of uncertainty among patients having peritoneal dialysis. Journal of Advanced Nursing, 2009; 65(8): 1664-1669.

19. MANTOVANI MF, et al. Protocolos clínicos na orientação de pessoas com doenças crônicas. Cogitare Enfermagem, $2015 ; 20(4): 821-828$.

20. MANTOVANI MF, et al. Depressão e qualidade de vida em adultos com hipertensão. Cogitare Enfermagem, 2017; 22(3); e51630.

21. MCDOWELL I. Measuring health: a guide to rating scales and questionnaires. 3nd ed. Oxford: University Press, 2006; $3 p$.

22. MENDES KDS, et al. Revisão integrativa: método de pesquisa para a incorporação de evidências na saúde e na enfermagem. Texto \& Contexto Enfermagem, 2008; 17(4): 758-764.

23. MULDER M, et al. Validation of a screening instrument for the fear of injection in dialysis patients. Journal of Renal Care, 2013; 39(4): 214-221.

24. OTTAVIANI AC, et al. Associação entre ansiedade e depressão e a qualidade de vida de pacientes renais crônicos em hemodiálise. Texto \& Contexto Enfermagem, 2016; 25(3): 1-8.

25. PAUDEL K, et al. . Lack of motivation: a new modifiable risk factor for peritonitis in patients undergoing peritoneal dialysis?. Journal of Renal Care, 2015; 41(1): 33-42.

26. RAJATI F, et al. Comparative evaluation of health-related quality of life questionnaires in patients with heart failure undergoing cardiac rehabilitation: a psychometric study. Archives of Physical Medicine and Rehabilitation, 2016; 97(11): 1953-1962.

27. RÔLA CVS, et al. Instrumentos de avaliação da qualidade de vida de pessoas jovens e idosas: um estudo de revisão sistemática. Revista Multidisciplinar de Psicologia, 2018; 12(42): 111-120.

28. SILVA ATM, et al. Escala preditiva de complicações da hipertensão: validade de conteúdo e aparência. Revista Mineira de Enfermagem, 2017; 21: e-1007.

29. SNAITH RP. The hospital anxiety and depression scale. Health and quality of life outcomes, 2003: 1(1): 1-4.

30. SOARES DA, et al. Qualidade de vida de portadores de insuficiência cardíaca. Acta Paulista de Enfermagem, 2008; $21(2): 243-248$.

31. SOUZA FTZ, OLIVEIRA JHA. Sintomas depressivos e ansiosos no paciente renal crônico em tratamento conservador. Revista Psicologia e Saúde, 2017; 9(3): 17-31.

32. SOUZA AR, et al. Fatores associados a um impacto na qualidade de vida pós-revascularização miocárdica. Revista da Rede de Enfermagem do Nordeste, 2018; 19: 1-10.

33. SUN V, et al. Symptom Concerns and Quality of Life in Hepatobiliary Cancers. Oncology Nursing Forum, 2018; 35(3): 45-52. 
34. TSAI SH, et al. Original Research The Efficacy of a Nurse-Led Breathing Training Program in Reducing Depressive Symptoms in Patients on Hemodialysis A Randomized Controlled Trial. AJN The American Journal of Nursing, 2015; 115(4): 24-32.

35. ULBRICH EM. Fatores preditivos para complicações em pessoas com hipertensão arterial sistêmica e ações para o gerenciamento de cuidados da enfermagem na atenção primária. Tese (Doutorado em Enfermagem) - Universidade Federal do Paraná, Curitiba, 2015; 173 p.

36. VILLAR RR, et al. Qualidade de vida e ansiedade em mulheres com câncer de mama antes e depois do tratamento. Revista Latino-Americana de Enfermagem, 2017; 25: 1-13.

37. VOORN VMA, et al. An innovative care model coordinated by a physical therapist and nurse practitioner for osteoarthritis of the hip and knee in specialist care: A prospective study. Rheumatology International, 2013; 33: 18211828.

38. WARE JE, SHERBOURNE CD. The MOS 36-Item Short-Form Health Survey (SF-36): Conceptual Framework and Item Selection. Medical Care, 1992; 30(6): 473-483.

39. WOOD-BAKER R, et al. Clinical trial of community nurse mentoring to improve self-management in patients with chronic obstructive pulmonary disease. International Journal Chronic Obstructive Pulmonary Disease, 2012; 7: 407413.

40. ZAKRISSON AB, et al. A three-year follow-up of a nurse-led multidisciplinary pulmonary rehabilitation programme in primary health care: a quasi-experimental study. Journal Clinic of Nursing, 2016; 25(7-8): 962-971. 